\title{
The white dwarf revealed in the intermediate polar V709 Cassiopeiae*
}

\author{
J. M. Bonnet-Bidaud ${ }^{1}$, M. Mouchet ${ }^{2,3}$, D. de Martino ${ }^{4}$, G. Matt ${ }^{5}$, and C. Motch ${ }^{6}$ \\ 1 Service d'Astrophysique, DSM/DAPNIA/SAp, CE Saclay, 91191 Gif-sur-Yvette Cedex, France \\ 2 DAEC et UMR 8631 du CNRS, Observatoire de Paris, Section de Meudon, 92195 Meudon Cedex, France \\ 3 Université Denis Diderot, Place Jussieu, 75005 Paris, France \\ e-mail: martine.mouchet@obspm.fr \\ ${ }^{4}$ Osservatorio Astronomico di Capodimonte, 80131 Napoli, Italy \\ e-mail: demartino@na.astro.it \\ 5 Dipartimento di Fisica, Universita Roma Tre, 00146 Roma, Italy \\ e-mail: matt@fis.uniroma3.it \\ ${ }^{6}$ CNRS, Observatoire de Strasbourg, 67000 Strasbourg, France \\ e-mail: motch@astro.u-strasbg.fr
}

Received 3 April 2001 / Accepted 23 May 2001

\begin{abstract}
Results are presented from the first detailed spectroscopic observations of the recently identified intermediate polar RX J0028.8+5917/V709 Cas. The study of the emission line radial velocities allows us to remove the uncertainties on the different aliases of the orbital period and a best value is found at $(0.2225 \pm 0.0002)$ day. It is also found that the system shows significant $E W \sim(2-4) \AA$ broad absorptions affecting the Balmer lines from $\mathrm{H}_{\delta}$ to $\mathrm{H}_{\beta}$. These broad absorptions are interpreted as the contribution of an underlying white dwarf atmosphere. The characteristics of the absorptions are found to be consistent with a DA $\log g=8$ white dwarf at a temperature of $\sim 23000 \mathrm{~K}$, contributing $\sim 17 \%$ (at $4500 \AA$ ) to the overall flux. This is the first direct detection of a white dwarf in an intermediate polar system. The absence of significant Zeeman splitting indicates a magnetic field lower than $10 \mathrm{MG}$, confirming that, at least in some cases, intermediate polars have weaker fields than polars. Different possibilities are discussed to explain the substantial contribution of the white dwarf to the overall flux.
\end{abstract}

Key words. stars: white dwarfs - stars: novae, cataclysmic variables - stars: magnetic fields X-rays stars: V709 cas

\section{Introduction}

Cataclysmic Variables (CVs) consist of a white dwarf accreting matter from a low mass companion filling its Roche lobe which, in some cases, can be an evolved secondary. Among them, polars and intermediate polars, or magnetic CVs, are a sub-class where the magnetic field of the white dwarf strongly affects the mass flow within the binary system (see reviews by Cropper 1990 and Patterson 1994). In polars (also called AM Her stars), the field is strong enough to lock the white dwarf spin in synchrony with the orbit and to control the flow into an accretion column. In Intermediate Polars (IP, previously DQ Her stars), the field is thought to be weaker, leading to a desynchronisation with a white dwarf spin faster than the orbit. The

Send offprint requests to: J. M. Bonnet-Bidaud, e-mail: bobi@discovery. saclay.cea.fr

* Based on data collected at the Haute-Provence Observatory OHP (France). accretion may then take place either through an accretion disc (disk-fed), or directly from the inner Lagrangian point (diskless) or via a combination of both (overflowdisk) (see review by Patterson 1994). Since CVs are interacting binary systems, their emission from the X-rays to the infrared is generally totally dominated by the release of the gravitational energy of the accreted matter in a disk and/or an accretion column and not by the emission of the stellar components (white dwarf and low-mass companion). In polars, some hints of the stellar characteristics can however be obtained during the period of low accretion rates which is a typical feature of these systems. During these low states, detected stellar photospheric lines can be used to constrain the temperature and magnetic field of the white dwarf (see Beuermann 1996; Sion 1999) as well as the nature and distance of the companion (see Cropper 1990; Warner 1995a). Possibly due to a different evolutionary stage and to a higher accretion rate, among IPs no such low states are known to exist and therefore 
very little is known about the stellar characteristics. The only cases where a stellar spectrum has been detected are for the very peculiar long period AE Aqr and GK Per systems where the secondary is an evolved star (Welsh et al. 1993; Reinsch 1994).

The X-ray source RX J0028.8+5917 was detected in the ROSAT All Sky Survey and identified as an intermediate polar on the basis of its spectral characteristics with a hard X-ray $(\sim 10 \mathrm{keV})$ component and a $N_{\mathrm{H}} \sim 10^{21}$ at $\mathrm{cm}^{-2}$ column density (Haberl \& Motch 1995). Further pointed observations by the ROSAT satellite led to the detection of an X-ray pulsation at $312.8 \mathrm{~s}$ with a $40 \%$ amplitude and a probable identification with coincident catalogued sources from previous different surveys $4 \mathrm{U} 0027+59,3 \mathrm{~A} 0026+593$ and $1 \mathrm{H} 0025+588$ (Haberl \& Motch 1995). Subsequent optical observations identified the source with the $m_{v} \sim 14$ variable star, V709 Cas, and line velocity variations reveal two possible orbital periods $P=5.4 \pm 0.2 \mathrm{~h}$ and $P=4.45 \pm 0.15 \mathrm{~h}$ (Motch et al. 1996, hereafter M96). Optical oscillations at the X-ray spin period and sideband have also been reported (Kozhevnikov 2001). A ROSAT observation in February 1998 showed a peculiar double-peaked X-ray pulsation with peaks separated by one third of the spin period and the source is suspected to be fed by accretion through a disk seen at low inclination angle and with a relatively weakly magnetized white dwarf (Norton et al. 1999). A more detailed spectral analysis has been provided by recent BeppoSAX and RXTE observations (de Martino et al. 2001a, submitted)

We report here on spectroscopic observations of the intermediate polar RX J0028.8+5917/V709 Cas, which clearly shows the evidence of broad Balmer absorption lines, superimposed on the usual IP emission line spectrum. These lines are attributed to the white dwarf atmosphere and are used to constrain for the first time the temperature and magnetic field of the white dwarf in an intermediate polar system.

\section{Observations and reduction}

Spectroscopic observations of V709 Cas were carried out at the $1.93 \mathrm{~m}$ telescope of the Haute-Provence Observatory (OHP), equipped with the Carelec spectrograph (Lemaitre et al. 1990). A $130 \AA / \mathrm{mm}$ grating was used to obtain low resolution $(F W H M \approx 7 \AA)$ spectroscopy over a wide $(3600-7200 \AA)$ range. Observations of (2-3) hour duration were performed in three consecutive nights and an additional one separated by one day (see Table 1). To avoid interruption in the orbital coverage, wavelength and photometric absolute calibrations were performed at the beginning and end of the run. A careful check for the stability of the wavelength calibration during the run was made, using the strongest sky emission lines present in the spectrum, such as the $\mathrm{O}$ [I] line $(5577.34 \AA)$, and small variations were corrected to an accuracy better than $0.1 \AA$. The standard stars $\mathrm{BD}+28^{\circ} 4211$ and Feige 110 were used for the photometric calibration on each night but due to variable atmospheric
Table 1. Log of observations.

\begin{tabular}{cccrc}
\hline Date & $T_{\text {start }}(*)$ & $T_{\text {end }}\left(^{*}\right)$ & $n$ & $T(\min )$ \\
\hline & & & & \\
1998 Aug. 21 & 1047.47164 & 1047.64034 & 9 & 20 \\
1998 Aug. 22 & 1048.42757 & 1048.64590 & 11 & 20 \\
1998 Aug. 23 & 1049.49154 & 1049.64089 & 8 & 20 \\
1998 Aug. 25 & 1051.45438 & 1051.64903 & 15 & 15 \\
\hline
\end{tabular}

(*) HJD(2450000+), $n$ number of spectra, $T$ exposure times.

absorption, only the night of Aug. 23 could be used to provide reliable absolute fluxes. To smooth out the influence of a possible modulation with the short $(\sim 5 \mathrm{~min})$ spin period, spectra were accumulated in either $15 \mathrm{~min}$ or $20 \mathrm{~min}$ exposures. All spectra were corrected for bias, flat-fielded and wavelength calibrated using the standard ESO/MIDAS procedures.

\section{Analysis and results}

\subsection{The two-component spectrum}

A total of 43 spectra were acquired over the 4 nights of observations. The spectra on the different nights are similar to those reported from the first optical observations by M96, showing strong Balmer and HeII (4686 $\AA$ ) lines in emission, superimposed on a blue continuum. Figure 1 shows a representative mean spectrum, averaged over one night. Weaker HeI and HeII emission lines are also visible in the figure. The bump in the continuum, visible around $5500 \AA$, is due to broad variable atmospheric extinction due to ozone, not corrected by the applied standard extinction curve. Absorptions around $6280 \AA$ and $6880 \AA$ are also atmospheric features. Absorption on the red side of $\mathrm{HeI}(5875 \AA)$ is most likely due to the $\mathrm{NaD}$ doublet. Its origin may be interstellar but it may also reveal a significant contribution from the secondary.

The overall spectrum of V709 Cas is typical of IPs but a more careful inspection of the lines reveals an unusual feature. Beside the presence of an emission component, the Balmer lines show a very significant symmetrical depression on both sides of the emission, suggestive of a broad absorption. This feature is absent in the HeII (4686 $\AA$ ) line and the $\mathrm{H}_{\alpha}$ line. The spectra obtained during the different nights all show the same characteristics, though it is slightly more pronounced on Aug. 23. The normalized source spectrum is presented in Fig. 2, which shows more clearly that the most significant absorption is seen at $\mathrm{H}_{\gamma}$ and $\mathrm{H}_{\delta}$. The feature is less symmetric around $\mathrm{H}_{\beta}$ where the blue part of the absorption seems to be filled up.

\subsection{The orbital period}

To take into account the absorption features, the line characteristics (velocities, equivalent widths), have been derived by a fit with a two-Gaussian function for emission and absorption, with the adjacent continuum being 


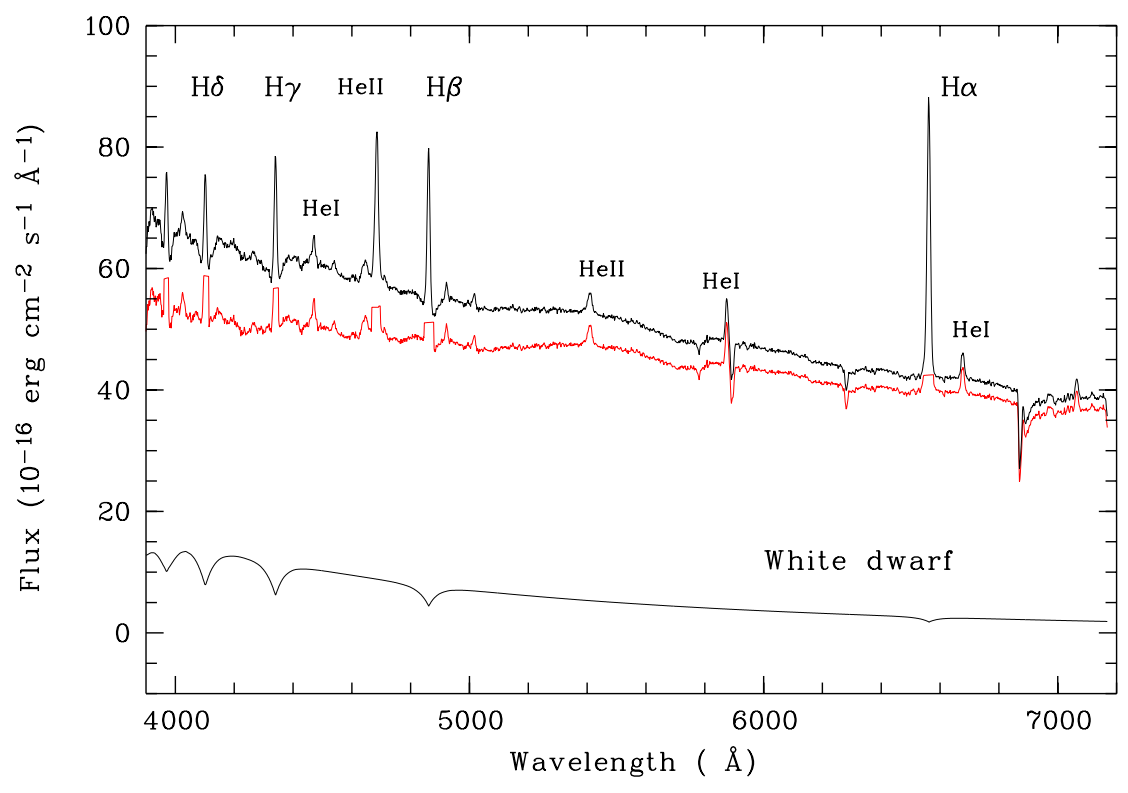

Fig. 1. The mean spectrum of V709 Cas on Aug. 23. The top curve is the observed spectrum showing a significant wide absorption affecting the Balmer lines from $\mathrm{H}_{\delta}$ to $\mathrm{H}_{\beta}$. The middle curve is the residual flux after subtracting a contribution from a $T=23000 \mathrm{~K}$ white dwarf whose flux distribution is shown by the bottom curve. Emission lines in the middle curve have been cut for clarity. The spectra have not been corrected for the telluric absorption lines. The narrow absorptions and the broad bump around $5500 \AA$ are atmospheric. The narrow absorption feature in the red side of HeI ( $5875 \AA)$ is identified as $\mathrm{NaD}$ (see text).
Table 2. Emission line radial velocities.

\begin{tabular}{lrrrr}
\hline Line & $\gamma\left(\mathrm{km} \mathrm{s}^{-1}\right)$ & $K\left(\mathrm{~km} \mathrm{~s}^{-1}\right)$ & \multicolumn{1}{c}{$\phi_{0}$} & \multicolumn{1}{c}{$\chi_{\mathrm{r}}^{2}$} \\
\hline & & & & \\
$\mathrm{H}_{\alpha}$ & $-64.7(1.5)$ & $102.6(2.3)$ & $0.000(3)$ & 4.0 \\
$\mathrm{H}_{\beta}$ & $-56.7(1.5)$ & $92.7(2.3)$ & $-0.022(4)$ & 4.0 \\
$\mathrm{H}_{\gamma}$ & $-37.6(1.5)$ & $88.9(2.3)$ & $-0.024(4)$ & 5.8 \\
$\mathrm{H}_{\delta}$ & $-51.0(1.5)$ & $77.1(2.3)$ & $-0.008(4)$ & 12.8 \\
$\mathrm{HeII}(4686 \AA)$ & $-91.1(1.5)$ & $75.5(2.3)$ & $+0.044(4)$ & 7.2 \\
\hline
\end{tabular}

Errors on last digit in parentheses.

described by a one degree polynomial. The velocities of the strongest $\mathrm{H}_{\alpha}$ emission line, measured over the 5 day interval, have been used to determine the orbital period. A Fourier transform (FFT) of the velocities was first used to constrain the period. The FFT yields two prominent peaks at periods $P=5.34 \pm 0.05 \mathrm{~h}$ and $P=4.37 \pm 0.06 \mathrm{~h}$ with smaller peaks at $P=3.70 \pm 0.06 \mathrm{~h}$ and $P=6.87 \pm 0.06 \mathrm{~h}$, all one day aliases with respect to each other, the errors being determined by the width of the peak at half maximum. The two most significant periods are in accordance with the values reported by M96.

Velocities of the emission lines were then folded with the two most significant periods and fitted with a function $V=\gamma+K \cdot \sin \left[(2 \pi \mathrm{t} / \mathrm{P})-\phi_{0}\right]$ with $\gamma, K$, and $\phi_{0}$ as free parameters, using a $\chi^{2}$ minimization program. For all lines, the fit is significantly better at $P=5.34 \mathrm{~h}$, so that the main alias period of $P=4.37 \mathrm{~h}$ can be securely rejected. For $\mathrm{H}_{\alpha}$, the reduced $\chi_{r}^{2}$ value decreases from $9.8(P=4.37 \mathrm{~h})$ to $4.0(P=5.34 \mathrm{~h})$, corresponding to a significance at a level better than $99 \%$, computed from a F-test. The $(O-C)$ values of the sine blueto-red crossing times, computed with the trial period of $5.34 \mathrm{~h}$ period, were then fitted to yield a best period value

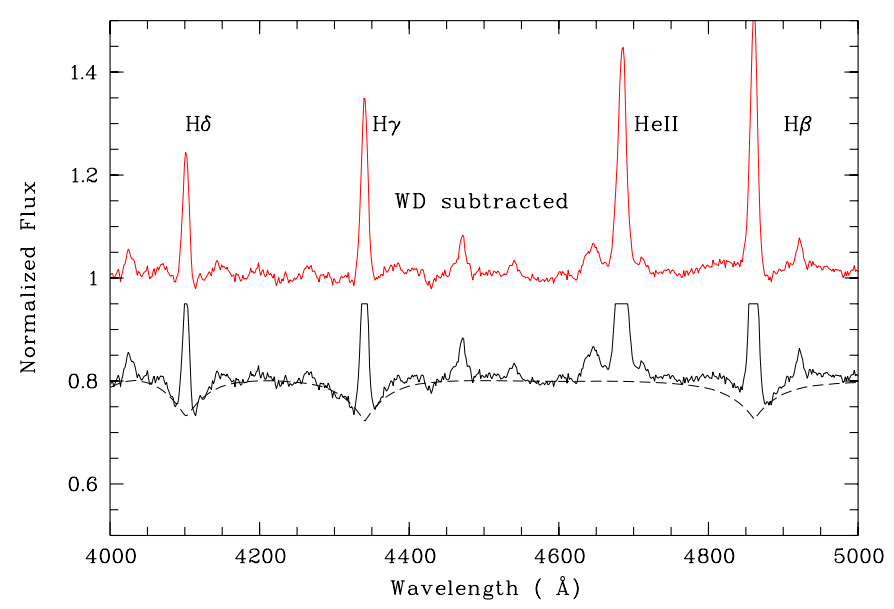

Fig. 2. A close-up of the average normalized spectrum of V709 Cas showing clearly the absorptions around $\mathrm{H}_{\delta}, \mathrm{H}_{\gamma}$ and $\mathrm{H}_{\beta}$ (lower curve, shifted by -0.2 unit for clarity). Note the absence of such a feature around HeII (4686 $\AA$ ). The dotted line shows the contribution of the best fit white dwarf (see text). The upper curve is the residual after subtraction of the white dwarf contribution.

of $P=(5.341 \pm 0.005)$ h with a corresponding ephemeris as $T_{\mathrm{o}}=$ HJD2451048.0575(2) $+E * 0.2225(2) \mathrm{d}$, where $T_{\mathrm{o}}$ is the blue-to-red crossing time of the emission lines.

\subsection{The line characteristics}

Table 2 gives the amplitude $(K)$ and phasing $\left(\phi_{0}\right)$ of the different emission lines folded with the above ephemeris. All Balmer lines show velocity curves with similar phasing but with radial velocity amplitudes decreasing through the Balmer series from $103 \mathrm{~km} \mathrm{~s}^{-1}\left(\mathrm{H}_{\alpha}\right)$ to $77 \mathrm{~km} \mathrm{~s}^{-1}$ $\left(\mathrm{H}_{\delta}\right)$. The HeII $(4686 \AA)$ shows the lowest amplitude at $\sim 76 \mathrm{~km} \mathrm{~s}^{-1}$ with a slight shift in phase and a different $\gamma$ velocity. 


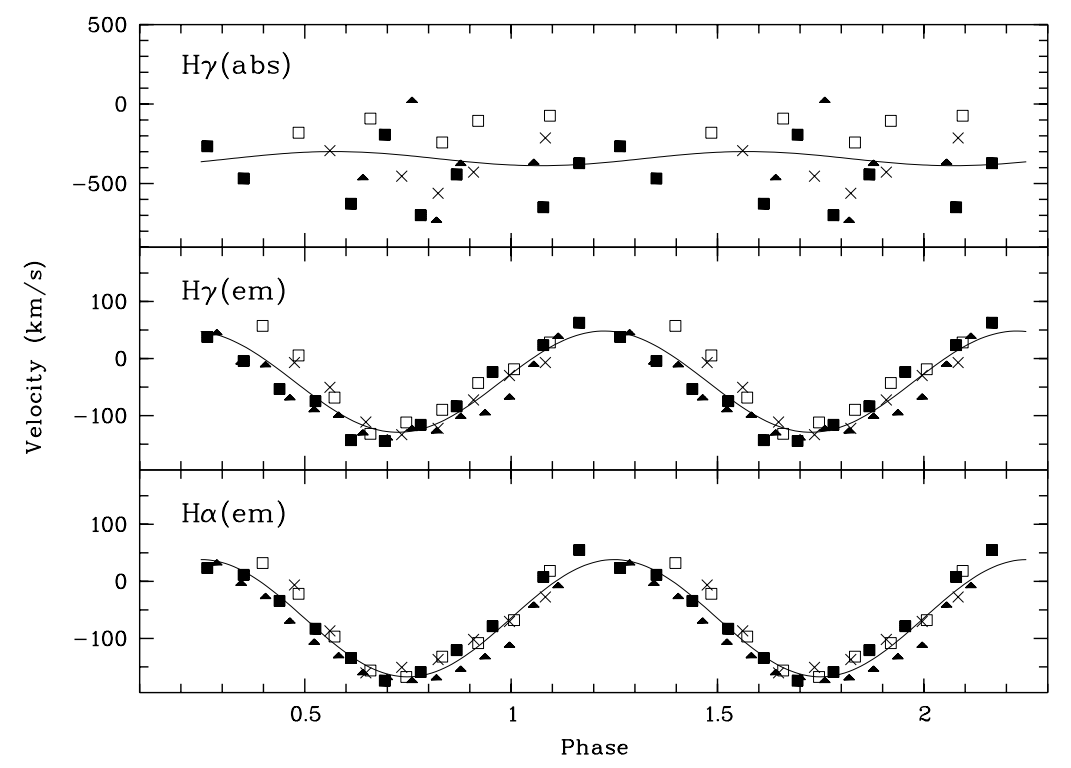

Fig. 3. Radial velocity curves as a function of orbital phase for the $\mathrm{H}_{\alpha}$ (bottom) and $\mathrm{H}_{\gamma}$ (middle) emission components and the $\mathrm{H}_{\gamma}$ (top) absorption component. The different nights are shown by different symbols (Aug. 21-squares, Aug. 22-filled squares, Aug. 23-crosses, Aug. 25filled triangles) and the best sinusoidal fits are shown by thin lines. Typical errors are $(3-5) \mathrm{km} \mathrm{s}^{-1}$ (bottom), (5-10) $\mathrm{km} \mathrm{s}^{-1}$ (middle) and (100-200) $\mathrm{km} \mathrm{s}^{-1}$ (top).

The velocity curves for $\mathrm{H} \alpha$ and $\mathrm{H} \gamma$ emission lines are shown in Fig. 3. Inspection of the curves reveals a significant dispersion of the points obtained on the different nights. Although the minimum around $\phi \sim 0.75$ is relatively well defined, there is a noticeable shift in velocity from one night to another for points at the same phase, in the range $\phi \sim(0.3-0.5)$ and (0.8-1.0). The dispersion from a pure sine curve is also reflected by the high values of the reduced $\chi_{\mathrm{r}}^{2}$ of the fits (Table 2). Using the sky emission lines, we checked that between the different nights the accuracy of the absolute calibration was better than $0.1 \AA$. The shift in velocity from night to night is of the order of $70 \mathrm{~km} \mathrm{~s}^{-1}\left(1.0 \AA\right.$ at $\mathrm{H}_{\gamma}$ and $1.5 \AA$ at $\left.\mathrm{H}_{\alpha}\right)$. There is no clear change in the line profiles from night to night but we cannot exclude the existence of some asymmetry in the lines that would be poorly fitted by Gaussian curves. Fits of the emission lines with an unique Gaussian function yield a similar amount of dispersion, showing that the effect is not due to the existence of the absorption component. The distortion is also present in $\mathrm{H}_{\alpha}$ where no absorption is seen. The influence of the spin period can also be excluded as the exposure times of 20 min and 15 min were chosen to be close multiples of the spin period (3.8 and 2.9 respectively). Possible significances of this dispersion are considered in the discussion.

The velocity of the $\mathrm{H}_{\gamma}$ absorption component, as deduced from the fits, is also plotted in Fig. 3. The measured values are however highly uncertain due to the very shallow and broad nature of the line and no significant radial velocity curve can be derived. An attempt was made to measure the velocity changes with a cross-correlation method, using one of the best $\mathrm{S} / \mathrm{N}$ spectra as a template. Though somewhat reducing the dispersion of the points, this method also does not reveal any regular variations.

Table 3 gives the measured equivalent widths $(E W)$ and intrinsic widths ( $F W$ at half-maximum) of the emis-
Table 3. Line full widths at half maximum $(F W)$ and equivalent widths $(E W)$.

\begin{tabular}{lrrrr}
\hline Line & \multicolumn{2}{c}{ Emission } & \multicolumn{2}{c}{ Absorption } \\
& $E W_{\mathrm{e}}(\AA)$ & $F W_{\mathrm{e}}(\AA)$ & $E W_{\mathrm{a}}(\AA)$ & $F W_{\mathrm{a}}(\AA)$ \\
\hline $\mathrm{H}_{\alpha}$ & $18.0(4)$ & $14(1)$ & - & - \\
$\mathrm{H}_{\beta}$ & $6.6(3)$ & $12(1)$ & $1.5(0.2)$ & $36(5)$ \\
$\mathrm{H}_{\gamma}$ & $4.7(3)$ & $11(1)$ & $3.5(0.1)$ & $58(7)$ \\
$\mathrm{H}_{\delta}$ & $3.8(3)$ & $11(1)$ & $3.0(0.1)$ & $37(5)$ \\
$\mathrm{HeII}(4686 \AA)$ & $6.7(3)$ & $13(1)$ & - & - \\
\hline
\end{tabular}

Errors on last digit in parentheses.

sion and absorption components. For the Balmer lines where an absorption is measured, the absorption is always very broad $\left(F W H M \sim 2500-4000 \mathrm{~km} \mathrm{~s}^{-1}\right)$ and with an $E W$ nearly comparable to emission, with the exception of $\mathrm{H}_{\beta}$ for which the $E W$ is significantly smaller, due to the partial filling of the blue part of the absorption. The intrinsic widths of the absorption component $(\sim 40$ $60 \AA$ ) are similar to what is observed in a spectrum of a DA white dwarf, with Balmer lines in absorption.

\section{Discussion}

The optical spectrum of V709 Cas, with strong $(E W \sim 4-18 \AA)$ Balmer and HeII (4686 $\AA$ ) emission lines, is typical of IPs. However, it also shows evidence of broad ( FWHM $\sim 40-60 \AA$ ) absorptions which were not yet noticed in this source nor in any other IP. Balmer lines in absorption are sometimes seen among classical CVs, mainly in nova-like systems in high states and during dwarf nova eruptions, which suggests that they are formed in an optically thick accretion disk with a high mass transfer (see for instance La Dous 1994; Hessman 1986). Absorption 
lines have also been observed among dwarf novae in a very few systems where the accretion rate is low, as in GW Lib (Szkody et al. 2000) or with a high inclination, as in OY Car (Hessman et al. 1989). The other known cases are among polars during low states where Balmer absorption lines are clearly seen, significantly split by the Zeeman effect. They are attributed in this case to the atmosphere of a strongly magnetized white dwarf (see Cropper 1990). In the case of V709 Cas, the stability of the optical flux excludes a dwarf nova event and points toward a white dwarf rather than a disk origin. The absence of He absorptions also does not favour a disk origin. The lack of any significant absorption around $\mathrm{H}_{\alpha}$ is indirect evidence that the spectral component responsible for the absorption has a rather steep continuum, contributing significantly only in the blue and becoming negligible in the red part of the spectrum by a dilution effect with the rest of the emission. This is therefore indicative of a hot white dwarf as commonly seen among polars.

We therefore attempted to describe the underlying absorption spectrum, using a grid of WD models with LTE atmospheres (Finley et al. 1997; Koester 2000, private communication). As the contribution of the white dwarf to the overall flux is unknown, we used an indirect method. The temperature-gravity domain was first constrained by comparing the observed intrinsic line widths with the ones predicted from theoretical models. The contribution of the white dwarf to the overall flux was then deduced from the measured $E W$ of the absorption lines.

For the determination of the observed parameters, we use primarily the $\mathrm{H}_{\gamma}$ measurements as the $\mathrm{H}_{\delta}$ line is weaker and the $\mathrm{H}_{\beta}$ line seems to be contaminated by side emissions. Figure 4 shows the theoretical FWHM for a grid of LTE models of DA white dwarfs with a temperature ranging from $T=15000$ to $45000 \mathrm{~K}$ and a gravity from $\log g=7.5$ to 8.5. Assuming a gravity of $\log g=8$, the measured $F W H M$ given in Table 3 is consistent with a temperature in the range $T=18000-30000 \mathrm{~K}$ with a best value at $23000 \mathrm{~K}$. If one allows for a full range of gravities, the $T-\log g$ domain is not unambiguously determined and the temperature is only weakly constrained in the range $15000-35000 \mathrm{~K}$.

A dilution factor was defined as the ratio of the white dwarf emission to the global continuum flux, $\alpha=$ $F_{\text {wd }} / F_{\text {cont }}$. For a continuum flux close to an emission line, $\alpha$ is also the ratio $E W_{\text {abs }} / E W_{\mathrm{th}}$, where $E W_{\mathrm{abs}}$ is the observed $E W$ of the absorption (including dilution) and $E W_{\text {th }}$ the theoretical $E W$ value. The dilution factor and hence the contribution of the white dwarf to the global flux can then be evaluated from the line $E W$. Assuming $\log g=8$, the measured $E W$ of the $\mathrm{H}_{\gamma}$ line in Table 3 yields a dilution factor of $(0.17 \pm 0.04)$ at $\sim 4500 \AA$ for the range of temperatures determined above. Given the steep energy distribution of the white dwarf, its contribution is significantly lower around $\mathrm{H}_{\alpha}$ with $\alpha \sim 0.06$, corresponding to an expected absorption component of $E W_{\text {abs }} \sim 1 \AA$. This is negligible compared to the emis-

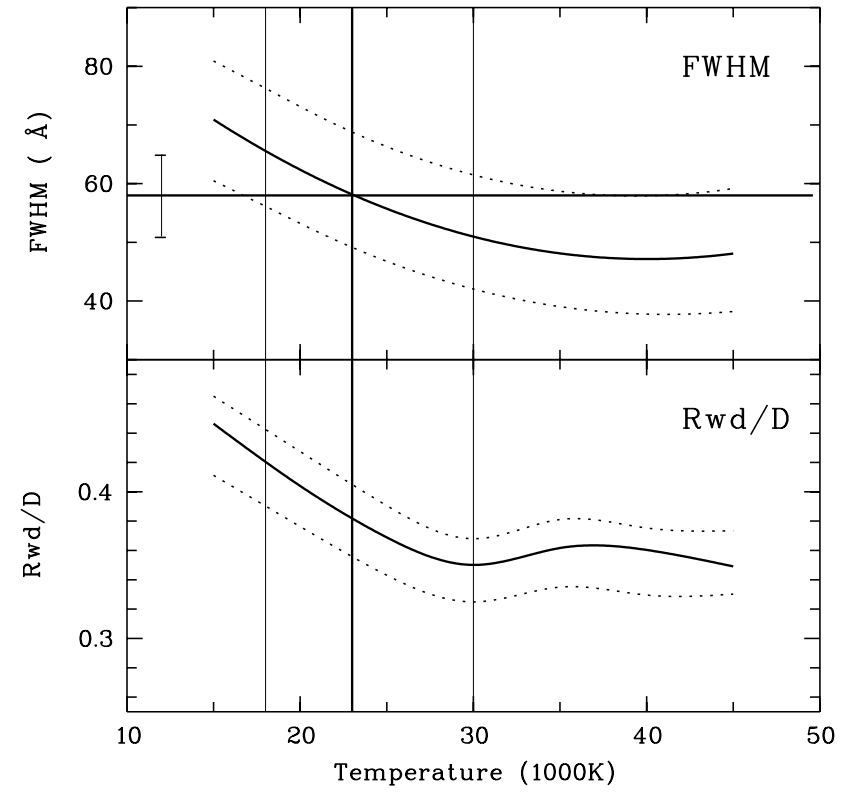

Fig. 4. Upper panel: The theoretical $\mathrm{H}_{\gamma} F W H M$ values predicted from white dwarf atmospheric models for a range of temperatures and different $\log g$ (log $g=7.5$ upper dotted, 8.0 middle thick, 8.5 lower dotted). The range of values consistent with the measured $F W H M$ absorptions (51-65 $\AA$ ) and $\log g=8.0$ are shown by vertical lines. Lower panel: the $R_{\mathrm{wd}} / D$ values, in units of $10^{9} \mathrm{~cm}$ for $R_{\mathrm{wd}}$ and $100 \mathrm{pc}$ for $D$, as derived from a comparison of the observed and theoretical flux (see text) for the same parameter range.

sion component $E W_{\mathrm{em}} \sim 18 \AA$, therefore explaining the absence of noticeable absorption at $\mathrm{H}_{\alpha}$.

The comparison of the observed WD flux with the predicted theoretical flux yields then an additional constraint on the $R_{\mathrm{wd}} / D$ ratio shown in Fig. 4, where $R_{\mathrm{wd}}$ is the white dwarf radius in units of $10^{9} \mathrm{~cm}$ and $D$ is the system distance in units of 100 pc. For $\log g=8.0$, $R_{\mathrm{wd}}$ is $\sim 0.87 \times 10^{9} \mathrm{~cm}$ for a standard M/R relation (Nauenberg 1972) and, from the range of temperatures $(T=18000-30000 \mathrm{~K})$ consistent with the observed $F W H M$, the system distance is then derived as $D=$ (210-250) pc.

The assumption of an underlying WD spectrum responsible for the observed broad absorption lines leads therefore to a $T \sim 23000 \mathrm{~K}$ white dwarf contributing $\sim 17 \%$ (around $4500 \AA$ ) to the overall flux for a system at $\sim 230$ pc. These values are quite consistent with what can be expected from a typical IP system. The absence of absorption associated with the He lines suggests a pure DA white dwarf, at the difference of a disk emission where absorption helium lines could be produced. Another interesting feature is the absence of Zeeman splitting, such as is observed in polars during low states. A significant Zeeman splitting is expected and observed in isolated white dwarfs for magnetic fields greater than 6-10 MG (see Achilleos et al. 1991) and is commonly present in polar systems with $B$ fields in the range 10-30 MG. Such components are not detected here for $\mathrm{H}_{\beta}$ or $\mathrm{H}_{\gamma}$. With the same method as 
described in Bonnet-Bidaud et al. (2000), synthetic profiles have been built using the Zeeman wavelengths and oscillator strengths tabulated by Kemic (1974). The absorption features expected at the position of the Zeeman components from a magnetic field as low as 3 MG have been compared to the observed spectra. We find in particular that for the lowest tabulated $B$ value of $3 \mathrm{MG}$, the $\mathrm{H}_{\beta} \sigma$ components at $4832 \AA$ and $4896 \AA$ are clearly missing. The observations are therefore the first direct indication that, at least in some intermediate polars, the white dwarfs have lower magnetic fields than in polars. A low magnetic field for V709 Cas seems also suggested by the short $(312 \mathrm{~s})$ spin period of the system if one assumes that the system is at equilibrium with a spin period corresponding to the Keplerian period at the Alfvén radius (see Norton et al. 1999).

The question arises as to why V709 Cas is, so far, the only system among IPs where the white dwarf is seen to contribute significantly to the overall flux so that the atmospheric absorptions become visible. From the deduced best characteristics, there is no indication that the white dwarf is atypical. Its temperature $(T \sim 23000 \mathrm{~K})$ is similar, though at the upper edge of the values reported for magnetic CVs (Sion 1999). However, the gravity being not fully constrained; the contribution of the white dwarf could be indeed more important if the gravity is somewhat lower. This would imply a larger radius together with a higher temperature, as seen from Fig. 4. For $\log g=7.5$, the white dwarf contribution around $4500 \AA$ would be larger by a factor $\sim 3.5$ when compared to $\log g=8.0$. This would however also correspond to a rather low mass object $\left(\sim 0.34 M_{\odot}\right)$ with a very peculiar high temperature $(T \sim 35000 \mathrm{~K})$, the lower limit being at $T \geq 25000 \mathrm{~K}$ from the lower bound of the $F W H M$ values.

A sufficient contrast between the white dwarf and the overall flux could also be obtained if the contributions from the other regions in the system are significantly lower when compared to other intermediate polars. From the residual (total-white dwarf) flux at a distance of $\sim 230 \mathrm{pc}$, an absolute magnitude of $M_{v} \sim 7.6$ is derived, after correction for a visual extinction of $A_{V} \sim 0.45$ corresponding to the $N_{\mathrm{H}} \sim 8.7 \times 10^{20}$ at $\mathrm{cm}^{-2}$ column density observed in the X-rays (de Martino et al. 2001a, submitted). If attributed to a disk, and using the crude $\left(M_{v}-\dot{M}\right)$ relation given in Smak (1989) for face-on blackbody disks, this absolute magnitude would correspond, for a $\sim 0.6 M_{\odot}(\log g=8.0)$ white dwarf, to a $\dot{M}$ in the range $\sim(1-2) \times 10^{16} \mathrm{~g} \mathrm{~s}^{-1}$, depending on the exact extension of the disk. This is consistent with the range of values $\sim(0.7-1.3) \times 10^{16} \mathrm{~g} \mathrm{~s}^{-1}$ derived from the recent $\mathrm{X}$ ray spectral analysis (de Martino et al. 2001a, submitted). If, as suggested from an analysis of the X-ray pulsations (Norton et al. 1999), the disk is seen at a relatively low inclination angle, its luminosity would therefore not be abnormaly low.
However, the possibility of a larger inclination angle cannot yet be excluded. The large dispersion seen in the radial velocity curves (see Sect. 3.3) is strongly suggestive of the S-wave line distorsions introduced by a variable hot spot contribution and/or partial eclipses of the line regions found in high inclination systems (see Warner 1995b). There is also some evidence from recent photometric observations of a possible partial disk eclipse (de Martino et al. 2001b, in preparation). We therefore cannot exclude that the important contribution of the white dwarf to the overall flux could be due to a combination of a low gravity white dwarf with a somewhat inclined disk.

Additional observations are also clearly needed to see if the visibility of the white dwarf also depends on the overall accretion rate and luminosity of the source.

Acknowledgements. We are very grateful to D. Koester and N. Dolez for having provided us with detailed catalogues of theoretical white dwarf spectra.

\section{References}

Achilleos, N., Remillard, R. A., \& Wickramasinghe, D. T. 1991, MNRAS, 253, 522

Beuermann, K. 1996, in Compact Stars in Binaries, ed. van Paradijs, 403

Bonnet-Bidaud, J. M., Mouchet, M., Shakhovskoy, N. M., et al. 2000, A\&A, 354, 1003

Cropper, M. 1990, Space Sci. Rev., 54, 195

de Martino, D., Matt, G., Belloni, T., et al. 2001a, A\&A, submitted

Finley, D. S., Koester, D., \& Basri, G. 1997, ApJ, 488, 375

Haberl, F., \& Motch, C. 1995, A\&A, 297, 37

Hessman, F. V. 1986, ApJ, 300, 794

Hessman, F. V., Koester, D., Schoembs, R., \& Parwigh, H. 1989, A\&A, 213, 167

Kozhevnikov, V. P. 2001, A\&A, 366, 891

La Dous, C. 1994, Sp. Sci. Rev., 67, 1

Lemaitre, G., Kohler, D., Lacroix, D., Meunier, J. P., \& Vin, A. 1990, A\&A, 228, 546

Motch, C., Haberl, F., Guillout, P., et al. 1996, A\&A, 307, 459 [M96]

Nauenberg, M. 1972, ApJ, 175, 417

Patterson, J. 1994, PASP, 106, 209

Norton, A. J., Beardmore, A. P., Allan, A., \& Hellier, C. 1999, A\&A, 347, 203

Reinsch, K. 1994, A\&A, 281, 108

Sion, E. 1999, PASP, 111, 532

Smak, J. 1989, Acta Astron., 39, 317

Szkody, P., Desau, V., \& Hoard, D. W. 2000, AJ, 119, 365

Warner, B. 1995a, Ap. Sp. Sci., 232, 89

Warner, B. 1995b, in Cataclysmic Variables Stars (Cambridge Univ. Press)

Welsh, W. F., Horne, K., \& Oke, J. B. 1993, ApJ, 406, 229 